\title{
Ground-state factorization and quantum phase transition in dimerized spin chains
}

\author{
Gian Luca Giorgi* \\ Dipartimento di Fisica, Università di Roma La Sapienza, Piazzale A. Moro 2, 00185 Roma, Italy
}

(Received 22 January 2009; published 11 February 2009)

\begin{abstract}
We study the occurrence of ground-state factorization in dimerized $X Y$ spin chains in a transverse field. Together with the usual ferromagnetic and antiferromagnetic regimes, a third case emerges, with no analogous in translationally invariant systems, consisting of an antiferromagnetic Neél-type ground state where pairs of spins represent the unitary cell. Then, we calculate the exact solution of the model and show that the factorizing field represent an accidental degeneracy point of the Hamiltonian. Finally, we extend the study of the existence of ground-state factorization to a more general class of models.
\end{abstract}

DOI: 10.1103/PhysRevB.79.060405

PACS number(s): 75.10.Jm, 03.67.Mn, 64.70.Tg

The study of zero-temperature critical phenomena in quantum magnetic systems represents since long time a major research subject. ${ }^{1-4}$ In particular, the $X Y$ model is a very rich source of information about the quantum behavior of spin chains because of the availability of an exact analytical solution. ${ }^{3,5}$ During the last years, the main interest about spin chains concerned the relationship between quantum phase transitions and entanglement. ${ }^{6-9}$ Among a number of interesting properties of such systems, it is worth citing the existence of special values of the external magnetic field, the parameter which drives the phase transition, which give rise to ground-state factorization, discovered by Kurmann et al. ${ }^{10}$ This phenomenon has been observed in two-dimensional lattices through quantum Monte Carlo methods ${ }^{11}$ and fully analyzed by Giampaolo et al. ${ }^{12}$ in a recent publication, where the factorizing field has been determined for a quite general class of models. They developed an appropriate measure of entanglement which vanishes at the factorizing point. So far, the existence of a factorized ground state has been predicted only in translationally invariant Hamiltonian models.

Moreover, critical properties of physical systems are discussed by taking the thermodynamic limit from the beginning. On the other hand, the knowledge of a finite-size solution clarifies important aspects of this limit. For example, it is known that the quantum phase diagram of the $X Y$ chain in a transverse field exhibits two different symmetry-broken regions characterized by different behaviors of two-body correlation functions. This dissimilarity has a microscopic origin easily understood in the finite-size case. Besides these considerations, the study of finite systems is relevant by itself for the realization of mesoscopic qubits of contemporary interest.

In this Rapid Communication we discuss a finite-size dimerized $X Y$ spin chain in a transverse field, and analyze ground-state properties. The interest about such a system, belonging to a more general class of models, ${ }^{13-15}$ is motivated by experimental work on quasicrystals and quasiperiodic superlattices. ${ }^{16,17}$ First of all, we show that the model admits the existence of a factorized ground state, and then we discuss the exact solution. The factorizing field turns out to be an accidental degeneracy point of the Hamiltonian and falls on a border surface between two regions that, in the thermodynamic limit, are characterized by different symmetry-breaking mechanisms. Furthermore, we will be able to detect the conditions for the existence of ground-state factorization also in a more general class of dimerized chains which are the generalization of the model discussed in Ref. 12.

We start our discussion by considering a nearest-neighbor dimerized chain of an even number $N$ of $\operatorname{spin} 1 / 2$ :

$$
\begin{aligned}
\mathcal{H}= & \sum_{l=1}^{N / 2} \sum_{i=1}^{2}\left[\frac{J_{i}+\gamma_{i}}{2} \sigma_{2 l-2+i}^{x} \sigma_{2 l-1+i}^{x}+\frac{J_{i}-\gamma_{i}}{2} \sigma_{2 l-2+i}^{y} \sigma_{2 l-1+i}^{y}\right] \\
& -h \sum_{l=1}^{N} \sigma_{l}^{z},
\end{aligned}
$$

with $\sigma_{N+1}^{\alpha}=\sigma_{1}^{\alpha}$, being $\sigma_{l}^{\alpha}$ the $\alpha$ th Pauli matrix $(\alpha=x, y, z)$. Without loss of generality, we will limit the analysis to positive fields. The Hamiltonian of Eq. (1) can be recast in the form of a sum over two-body Hamiltonians $\mathcal{H}=\sum_{l=1}^{N / 2}\left(\mathcal{H}_{l}^{(1)}\right.$ $\left.+\mathcal{H}_{l}^{(2)}\right)$, where

$$
\begin{aligned}
\mathcal{H}_{l}^{(i)}= & \frac{J_{i}+\gamma_{i}}{2} \sigma_{2 l-2+i}^{x} \sigma_{2 l-1+i}^{x}+\frac{J_{i}-\gamma_{i}}{2} \sigma_{2 l-2+i}^{y} \sigma_{2 l-1+i}^{y} \\
& -h_{i}\left(\sigma_{2 l-2+i}^{z}+\sigma_{2 l-1+i}^{z}\right),
\end{aligned}
$$

with $h_{1}$ and $h_{2}$ such that $h=h_{1}+h_{2}$.

The central feature of $\mathcal{H}_{l}^{(i)}$ is the invariance under rotations of $\pi$ around the $z$ axis. This is formalized by the vanishing of the commutator $\left[\mathcal{H}_{l}^{(i)}, \mathcal{P}_{l}^{(i)}\right]=0$, where $\mathcal{P}_{l}^{(i)}$ $=\sigma_{2 l-2+i}^{z} \sigma_{2 l-1+i}^{z}$ is the parity operator since its eigenvalues are +1 or -1 , according to the number of down spins in the $z$ direction being even or odd. The above commutation relation then requires also the eigenstates of $\mathcal{H}_{l}^{(i)}$ to have definite parity. The problem is to establish whether there exists a set of the Hamiltonian parameters such that the ground state is of the form $|\Psi\rangle=\otimes_{l}\left|\psi_{l}\right\rangle$. Notice that, if this is the case, as remarked in Ref. 10, $\left|\psi_{2 l-2+i}\right\rangle\left|\psi_{2 l-1+i}\right\rangle$ must be the ground state of $\mathcal{H}_{l}^{(i)}$. The problem is then reduced to find the conditions under which $\mathcal{H}_{l}^{(i)}$ admits a factorized ground state. Now, since $\mathcal{H}_{l}^{(i)}$ is not diagonal in the $\sigma^{z}$ basis, if $\left|\psi_{2 l-2+i}\right\rangle\left|\psi_{2 l-1+i}\right\rangle$ has to be the ground state, each factor must be of the form $\left|\psi_{l}\right\rangle=\cos \psi_{l}|\uparrow\rangle+\sin \psi_{l}|\downarrow\rangle$, with $\psi_{l} \neq 0, \pi / 2$. This, in turn, implies that in the factorized ground state the parity symmetry is broken and, therefore, looking for the condition on the parameters of the Hamiltonian leading to factorization of the ground state amounts to looking for the condition leading to the degeneracy of the even and odd 
lowest lying eigenstates without invoking the vanishing of entanglement indicators. ${ }^{12}$

There are three different physical scenarios to be considered: (i) both $J_{1}$ and $J_{2}$ are negative (ferromagnetic case); (ii) both $J_{1}$ and $J_{2}$ are positive (antiferromagnetic case); (iii) $J_{1}$ $>0$ and $J_{2}<0$ or vice versa (hybrid case). In any of these cases, factorization appears if and only if $\gamma_{1} / J_{1}=\gamma_{2} / J_{2}=\kappa$, i.e., only in the presence of perfect dimerization of the longitudinal part of $\mathcal{H}$, the factorized point falls in $h_{F}$ $=\left[\left(J_{1}+J_{2}\right) / 2\right] \sqrt{1-\gamma^{2}}$, and $\tan \psi_{2 l-1+i}= \pm\left[\left(1-\sqrt{1-\kappa^{2}}\right) / \kappa\right]^{1 / 2}$.

(i) In the ferromagnetic case, we find $\psi_{2 l-1+i}=\psi_{2 l-2+i}$. As expected, the factorized state is fully aligned along two possible directions: $|\Psi\rangle=\otimes_{l}\left|\psi_{l}^{ \pm}\right\rangle$.

(ii) In the second (antiferromagnetic) case, we find $\psi_{2 l-1+i}=-\psi_{2 l-2+i}$. Then, alternate directions for the spin determining a Neél-type ground state are observed: $|\Psi\rangle=\otimes_{l=0}^{(N / 2)-1}\left|\psi_{2 l+1}^{ \pm}\right\rangle\left|\psi_{2 l+2}^{+}\right\rangle$.

(iii) If both ferromagnetic and antiferromagnetic factorized ground states are of the same kind of those obtained in the homogeneous $X Y$ chain, ${ }^{10}$ the third (hybrid) case shows up an original character. Indeed, by assuming, for example, $J_{1}<0$ and $J_{2}>0$ we find the constraints $\psi_{2 l-1}=\psi_{2 l}$ and $\psi_{2 l}=-\psi_{2 l+1}$. As a consequence, the factorized ground state assumes the structure

$$
|\Psi\rangle=\otimes_{l=0}^{(N / 4)-1}\left|\psi_{4 l+1}^{ \pm}\right\rangle\left|\psi_{4 l+2}^{ \pm}\right\rangle\left|\psi_{4 l+3}^{\mp}\right\rangle\left|\psi_{4 l+4}^{\mp}\right\rangle .
$$

Thus, we obtain an antiferromagnetic Neél-type ground state, whose unitary cell is represented by a pair of spins. An additional requirement for the existence of the FP in this case is that $N / 4$ must be an integer number in order to avoid frustration effects.

Now, we discuss the general exact solution of Hamiltonian (1) and enlighten the role played by the factorizing field. The diagonalization method is given in Ref. 18. We discuss explicitly the finite-size limit. ${ }^{14,19}$ The first step is the introduction of the Jordan-Wigner transformation, mapping spins into spinless fermions, ${ }^{3}$ defined through $\sigma_{l}^{z}=1-2 c_{l}^{\dagger} c_{l}$, $\sigma_{l}^{+}=\prod_{j<l}\left(1-2 c_{l}^{\dagger} c_{l}\right) c_{l}$, and $\sigma_{l}^{-}=\prod_{j<l}\left(1-2 c_{l}^{\dagger} c_{l}\right) c_{l}^{\dagger}$, which leads to $\mathcal{H}=\mathcal{H}_{0}-\mathcal{P} \mathcal{H}_{1}$, with

$$
\begin{aligned}
\mathcal{H}_{0}= & \sum_{l=1}^{N / 2} \sum_{i=1}^{2}\left[J_{i}\left(c_{2 l-2+i}^{\dagger} c_{2 l-1+i}+\text { H.c. }\right)\right. \\
& \left.+\gamma_{i}\left(c_{2 l-2+i}^{\dagger} c_{2 l-1+i}^{\dagger}+\text { H.c. }\right)\right]-h \sum_{l=1}^{N}\left(1-2 c_{l}^{\dagger} c_{l}\right), \\
& \mathcal{H}_{1}=\left[J_{2}\left(c_{N}^{\dagger} c_{1}-c_{N} c_{1}^{\dagger}\right)+\gamma_{2}\left(c_{N}^{\dagger} c_{1}^{\dagger}-c_{N} c_{1}\right)\right],
\end{aligned}
$$

where the parity operator is $\mathcal{P}=\Pi_{l=1}^{N}\left(1-2 c_{l}^{\dagger} c_{l}\right)$. Since $[\mathcal{H}, \mathcal{P}]=0$, all eigenstates of $\mathcal{H}$ have definite parity, and we can proceed to a separate diagonalization of $\mathcal{H}$ in the two subspaces corresponding to $\mathcal{P}= \pm 1$. Then, the complete set of eigenvectors of $\mathcal{H}$ will be given by the odd eigenstates of $\mathcal{H}^{-}=\mathcal{H}_{0}+\mathcal{H}_{1}$ and the even eigenstates of $\mathcal{H}^{+}=\mathcal{H}_{0}-\mathcal{H}_{1}$. Both for $\mathcal{H}^{-}$and $\mathcal{H}^{+}$the diagonalization can be performed through the division of the lattice in two sublattices: $c_{2 l-1}=a_{l}$, and $c_{2 l}=b_{l}$, and with the help of two separate Fourier transforms $a_{l}=(N / 2)^{-1 / 2} \Sigma_{k} a_{k} \exp \left[-i \frac{4 \pi k l}{N}\right]$ and $b_{l}=(N / 2)^{-1 / 2} \Sigma_{k} b_{k} \exp$ $\left[-i \frac{4 \pi k l}{N}\right]$, where $k=0,1, \ldots(N / 2)-1 \quad$ in $\mathcal{H}^{-}$, and $k$ $=1 / 2,3 / 2, \ldots(N / 2)-1 / 2$ in $\mathcal{H}^{+}$, getting

$$
\begin{aligned}
\mathcal{H}^{ \pm}= & \sum_{k}\left[J_{k} a_{k}^{\dagger} b_{k}-J_{k}^{*} a_{k} b_{k}^{\dagger}+\gamma_{k} a_{k}^{\dagger} b_{-k}^{\dagger}-\gamma_{k}^{*} a_{k} b_{-k}\right] \\
& -h \sum_{k}\left(2-2 a_{k}^{\dagger} a_{k}-2 b_{k}^{\dagger} b_{k}\right),
\end{aligned}
$$

with $J_{k}=J_{1}+J_{2} \exp \left[-i \frac{4 \pi k l}{N}\right], \quad \gamma_{k}=\gamma_{1}-\gamma_{2} \exp \left[-i \frac{4 \pi k l}{N}\right]$. We remark that the difference between $\mathcal{H}^{+}$and $\mathcal{H}^{-}$consists in the different set of allowed values of $k$. Finally, we discuss a generalized Bogoliubov transformation connecting $a_{k}, a_{-k}^{\dagger}, b_{k}, b_{-k}^{\dagger}$, and obtain two different kinds of quasiparticles,

$$
\mathcal{H}^{ \pm}=\sum_{k} \Lambda_{k}^{(+)}\left(2 \eta_{k}^{\dagger} \eta_{k}-1\right)+\sum_{k} \Lambda_{k}^{(-)}\left(2 \xi_{k}^{\dagger} \xi_{k}-1\right)
$$

where the eigenvalues, belonging to two branches separated by an energy gap, are, for $k \neq 0, N / 4, \Lambda_{k}^{( \pm)}=\sqrt{r_{k} \pm \sqrt{s_{k}}}$, with $r_{k}=4 h^{2}+\left|J_{k}\right|^{2}+\left|\gamma_{k}\right|^{2}$ and $s_{k}=\left|J_{k}\right|^{2}\left(16 h^{2}+2\left|\gamma_{k}\right|^{2}\right)+J_{k}^{2} \gamma_{k}^{* 2}+\gamma_{k}^{2} J_{k}^{* 2}$, and

$$
\begin{aligned}
& \Lambda_{0}^{( \pm)}=\sqrt{4 h^{2}+\left(\gamma_{1}-\gamma_{2}\right)^{2}} \pm\left(J_{1}+J_{2}\right), \\
& \Lambda_{N / 4}^{( \pm)}=\sqrt{4 h^{2}+\left(\gamma_{1}+\gamma_{2}\right)^{2}} \pm\left(J_{1}-J_{2}\right) .
\end{aligned}
$$

Let us assume, for example, $\left(J_{1}+J_{2}\right)>0$ and $J_{1}>J_{2}$. Then, each $\Lambda_{k}$ is positive, with the exception of $\Lambda_{0}^{(-)}, \Lambda_{N / 4}^{(-)}$(both of them are eigenvalues of $\left.\mathcal{H}^{-}\right)$, which can assume negative values, respectively, for $h<h_{C}^{(1)}=\sqrt{\left(J_{1}-J_{2}\right)^{2}-\left(\gamma_{1}+\gamma_{2}\right)^{2}} / 2$ and for $h<h_{C}^{(2)}=\sqrt{\left(J_{1}+J_{2}\right)^{2}-\left(\gamma_{1}-\gamma_{2}\right)^{2}} / 2$. Let us assume also, without loss of generality, $h_{C}^{(1)}<h_{C}^{(2)}$. Due to the foregoing considerations, the ground state of $\mathcal{H}^{+}$is its vacuum, and the corresponding eigenvalue is $E_{0}^{+}=-\sum_{k=1 / 2}^{(N / 2)-(1 / 2)} \sum_{\nu=+}^{-} \Lambda_{k}^{(\nu)}$. As for $\mathcal{H}^{-}$, the lowest energy is $E_{0}^{-}=-\sum_{k=0}^{(N / 2)-1} \sum_{\nu=+}^{-}\left|\Lambda_{k}^{(\nu)}\right|$, while the ground-state structure depends on $h$. The ground state has two quasiparticles on modes $k=0, N / 4$ for $h<h_{C}^{(1)}$, one quasiparticle on the mode $k=0$ for $h_{C}^{(1)}<h<h_{C}^{(2)}$, and finally, it is the vacuum state for $h>h_{C}^{(2)}$.

In order to identify the ground state of $\mathcal{H}$, we must compare the lowest eigenvalue of $\mathcal{H}^{+}$belonging to an even eigenstate $\left(E_{0}^{\text {even }}\right)$ with the lowest eigenvalue of $\mathcal{H}^{-}$belonging to an odd eigenstate $\left(E_{0}^{\text {odd }}\right)$. Since the vacuum state is even, we immediately state that the lowest even eigenvalue of $\mathcal{H}$ is $E_{0}^{\text {even }}=E_{0}^{+}$. As far as the lowest odd eigenvalue of $\mathcal{H}$ is considered, only inside the region $\left\{h_{C}^{(1)}, h_{C}^{(2)}\right\}$, where there is one quasiparticle (odd number of excitations), $E_{0}^{\text {odd }}=E_{0}^{-}$. In fact, outside this region, $E_{0}^{-}$belongs to even eigenstates (vacuum or two-quasiparticle state), and we must look to the firstexcited state of $\mathcal{H}^{-}$. Thus, $E_{0}^{\text {odd }}\left(h>h_{C}^{(2)}\right)=E_{0}^{-}\left(h>h_{C}^{(2)}\right)+\Lambda_{0}^{(-)}$, and $E_{0}^{\text {odd }}\left(h<h_{C}^{(1)}\right)=E_{0}^{-}\left(h<h_{C}^{(1)}\right)+\Lambda_{N / 4}^{(-)}$.

In the thermodynamic limit, the sum over $k$ becomes an integral, and the vacuum energies of $\mathcal{H}^{+}$and $\mathcal{H}^{-}$are identical: $E_{0}^{+}=E_{0}^{-}$. Thus, for $h_{C}^{(1)}<h<h_{C}^{(2)}, E_{0}^{\text {odd }}=E_{0}^{\text {even }}$ and spontaneous symmetry breaking comes out. Outside this range, the ground state has definite parity, being the energy gap $\Delta E$ $=2 \Lambda_{0}^{(-)}$for $h \geq h_{C}^{(2)}$ and $\Delta E=2 \Lambda_{N / 4}^{(-)}$for $h \leq h_{C}^{(1)}$. Thus, although phase transitions take place only for macroscopic systems, the change in the energy sign of quasiparticles can 


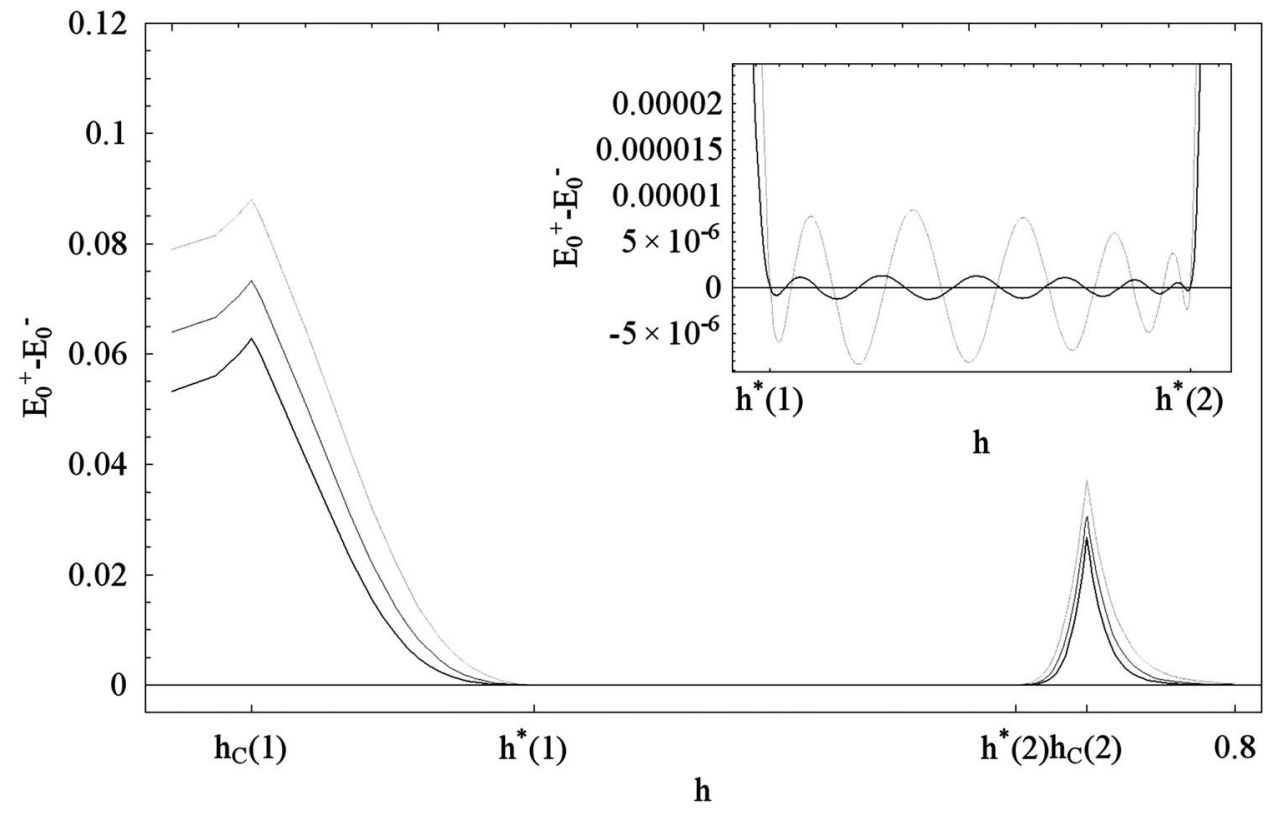

FIG. 1 . The energy difference $E_{0}^{+}-E_{0}^{-}$between the two ground states of $\mathcal{H}^{+}$and $\mathcal{H}^{-}$. Inside the range $\left\{h_{C}^{(1)}, h_{C}^{(2)}\right\}$ these two states are the lowest eigenstates of $\mathcal{H}$. The dimensionless Hamiltonian parameters are $J_{1}=1, J_{2}=0.4, \gamma_{1}=0.42, \gamma_{2}=0.168$. This choice is compatible with the existence of the factorized ground state $\left[h_{F}=h^{*}(2)\right]$, which is found to be around $h=0.63$. The other relevant parameters are $h_{C}^{(1)}$ $\simeq 0.06, h^{*}(1) \simeq 0.27$, and $h_{C}^{(2)} \simeq 0.69$. The light gray line refers to a chain of 20 spins and the gray line corresponds to 24 spin, while for the black curve a chain of 28 spin has been used. In the inset we plot detail of the bigger picture in the case $N=24$ (light gray) and $N=28$ (black) to enlighten the level crossing. As expected, we found $N / 2$ intersection points for each curve.

be used as a precursive property, allowing one to characterize the transition before performing the thermodynamic limit.

As in the homogeneous chain, ${ }^{5}$ in the presence of spontaneous symmetry breaking, two-body correlation functions can decrease monotonically or oscillate as a function of the spin distance depending on the Hamiltonian parameters. Recently, de Lima et al. ${ }^{14}$ found different regions in the phase diagram separated by hypersurfaces (collapsing into lines when $\left.\gamma_{1} / J_{1}=\gamma_{2} / J_{2}\right)$. We show that the first one of the separating lines corresponds to the factorizing field. Indeed, the different behavior of the correlation functions derives from different symmetry-breaking mechanisms. By analyzing lowest odd and even eigenvalues of $\mathcal{H}$ in the symmetry-broken region for any finite $N$ (Fig. 1), we observe a series of $N / 2$ intersection points $h_{i}$. The existence of such points has been discussed for the homogeneous chain in Refs. 20 and 21, and is responsible for magnetization jumps of Ref. 22. If $\gamma_{1} / J_{1}$ $=\gamma_{2} / J_{2}$, the first crossing point (for decreasing fields) is at $h=h_{F}$ for any $N$. In fact, $\quad \sum_{k=0}^{N / 2-1} \sum_{\nu=+}^{-} \Lambda_{k}^{(\nu)}\left(h_{F}\right)$ $=\sum_{k=1 / 2}^{N / 2-1 / 2} \sum_{\nu=+}^{-} \Lambda_{k}^{(\nu)}\left(h_{F}\right)=N\left(J_{1}+J_{2}\right)$. That is, $h=h_{F}$ is an accidental degeneracy point. If $\gamma_{1} / J_{1} \neq \gamma_{2} / J_{2}$, there are no fixed points. However, with the increasing of $N$, all the crossing points are confined inside the region limited by

$$
h^{*}(1)=\sqrt{\left(J_{1}-J_{2}\right)^{2}-\left(\gamma_{1}-\gamma_{2}\right)^{2}} / 2
$$

and

$$
h^{*}(2)=\sqrt{\left(J_{1}+J_{2}\right)^{2}-\left(\gamma_{1}+\gamma_{2}\right)^{2}} / 2 .
$$

These critical values define the separating surfaces of Ref. 14. It is worth noting that when a factorized field exists, $h_{F}$ $=h^{*}(2)$.
In the thermodynamic limit, this kind of structure implies two different symmetry-breaking mechanisms. As one can deduce from the results of Fig. 1, for $h^{*}(1)<h<h^{*}(2)$, as $N \rightarrow \infty$, the set $\left\{h_{i}\right\}$ of the degeneracy points becomes a denumerable infinity, i.e., an infinite number of crossing points appear and the two lower energies coincide, while for $h_{C}^{(1)}$ $<h<h^{*}(1)$ and for $h^{*}(2)<h<h_{C}^{(2)}$ there is the usual symmetry breaking due to the vanishing of the gap, i.e., the ground state has a definite parity for any finite $N$, but this difference goes to zero with $1 / N$. This is the microscopic mechanism responsible for dissimilar two-body correlation functions. Then, like in the homogeneous case, ${ }^{5}$ as the factorizing field is reached, correlation functions change character.

To conclude, we extend the study of ground-state factorization to a larger class of $X Y Z$ dimerized long-range spin models: $\mathcal{H}=\Sigma_{r} \mathcal{H}_{r}$, with

$$
\begin{aligned}
\mathcal{H}_{r}= & \sum_{l=1}^{(N / 2)-1}\left[J_{r, 1}^{x} \sigma_{2 l-1}^{x} \sigma_{2 l-1+r}^{x}+J_{r, 1}^{y} \sigma_{2 l-1}^{y} \sigma_{2 l-1+r}^{y}\right. \\
& \left.+J_{r, 1}^{z} \sigma_{2 l-1}^{z} \sigma_{2 l-1+r}^{z}\right]+\sum_{l=1}^{N / 2}\left[J_{r, 2}^{x} \sigma_{2 l}^{x} \sigma_{2 l+r}^{x}+J_{r, 2}^{y} \sigma_{2 l}^{y} \sigma_{2 l+r}^{y}\right. \\
& \left.+J_{r, 2}^{z} \sigma_{2 l}^{z} \sigma_{2 l+r}^{z}\right]-h \sum_{l=1}^{N} \sigma_{l}^{z},
\end{aligned}
$$

where $J_{r, i}^{\alpha}$ are the dimerized coupling constants between spin pairs at odd distance $r$. The existence of alternate coupling on even distances cannot be univocally introduced. However, we could consider homogeneous coupling for such distances. Notice that, assuming $J_{r, 1}^{\alpha}=J_{r, 2}^{\alpha}$ for any $r$, we recover the 
class of models considered by Giampaolo et al. in Ref. 12. For the sake of clarity, we will consider explicitly the case of a one-dimensional lattice, but the generalization to higher dimensions is straightforward.

Let us first note that, to circumvent frustration effects, $(N / r)$ has to be an integer number for any value of $r$ appearing in $\mathcal{H}$. As already done for the short-range $X Y$ chain, we rewrite $\mathcal{H}_{r}$ as a sum of two-body Hamiltonians, with

$$
\begin{aligned}
\mathcal{H}_{l, r}^{(i)}= & J_{r, i}^{x} \sigma_{2 l-2+i}^{x} \sigma_{2 l-2+i+r}^{x}+J_{r, i}^{y} \sigma_{2 l-2+i}^{y} \sigma_{2 l-2+i+r}^{y} \\
& +J_{r, i}^{z} \sigma_{2 l-2+i}^{z} \sigma_{2 l-2+i+r}^{z}-h_{i, r}\left(\sigma_{2 l-2+i}^{z}+\sigma_{2 l-2+i+r}^{z}\right),
\end{aligned}
$$

and $h_{1, r}, h_{2, r}$ such that $h=\sum_{r}\left(h_{1, r}+h_{2, r}\right)$.

The simplest case to study is the full ferromagnetic picture, where all coupling constants are negative. By following the same procedure introduced above, we proceed to calculate the ground-state energies for the two parity subspaces in each of two kinds of dimers $\left(\mathcal{H}_{l, r}^{(1)}\right.$ and $\left.\mathcal{H}_{l, r}^{(2)}\right)$, and force symmetry breaking. The factorized ground state of each of such dimers amounts to be $|\Psi\rangle_{l, r}^{(i)}=\left(\cos \psi_{r}^{(i)}|\uparrow\rangle \pm \sin \psi_{r}^{(i)}|\downarrow\rangle\right)_{l}$ $\otimes\left(\cos \psi_{r}^{(i)}|\uparrow\rangle \pm \sin \psi_{r}^{(i)}|\downarrow\rangle\right)_{l+r}$, with

$$
\tan ^{2} \psi_{r}^{(i)}=\frac{J_{r, i}^{x}+J_{r, i}^{y}-2 J_{r, i}^{z}+2 \sqrt{\left(J_{r, i}^{z}-J_{r, i}^{x}\right)\left(J_{r, i}^{z}-J_{r, i}^{y}\right)}}{J_{r, i}^{x}-J_{r, i}^{y}} .
$$

The existence of a globally factorized ground state implies that the angle $\psi_{r}^{(i)}$ must be the same for any of the dimers involving each site, i.e., it has to be independent both on $r$ and $i$. This result is achieved by the following conditions: $J_{r, 2}^{\alpha}=\kappa J_{r, 1}^{\alpha}$, and $J_{r, i}^{\alpha}=\gamma_{r} J_{1, i}^{\alpha}$. The value of the factorizing field is then $h_{F}=(1+\kappa) \sqrt{\left(\mathcal{J}_{1}^{z}-\mathcal{J}_{1}^{x}\right)\left(\mathcal{J}_{1}^{z}-\mathcal{J}_{1}^{\prime}\right)}$, where the $\mathcal{J}^{\alpha}$ are the global interactions along different axes: $\mathcal{J}_{i}^{\alpha}=\Sigma_{r} J_{r, i}^{\alpha}$.

For the full antiferromagnetic case, let first assume the nearest-neighbor interaction parameters such that $\left(J_{1, i}^{x}+J_{1, i}^{y}\right)$
$>0$. Then, we expect for the factorized ground state the structure $|\Psi\rangle=\otimes_{l=0}^{N / 2-1}\left|\psi_{2 l+1}^{ \pm}\right\rangle\left|\psi_{2 l+2}^{\mp}\right\rangle$. The range-dependent coupling constants have to be compatible with the existence of this state. This is possible only if $\left(J_{r, i}^{x}+J_{r, i}^{y}\right)>0$ for any $r$. The conditions that ensure the existence of a factorized ground state are $J_{r, 2}^{\alpha}=\kappa J_{r, 1}^{\alpha}$, and $J_{r, i}^{\alpha}=\gamma_{r} J_{1, i}^{\alpha}$, and the factorizing field amounts to $h_{F}=(1+\kappa) \sqrt{\left(\mathcal{F}_{1}+\mathcal{J}_{1}^{x}\right)\left(\mathcal{F}_{1}^{z}+\mathcal{J}_{1}^{y}\right)}$, with $\mathcal{J}_{1}^{\alpha}=\Sigma_{r} J_{r, 1}^{\alpha}$.

The second, hybrid, way to introduce antiferromagnetism is to fix, for example, $\left(J_{1,1}^{x}+J_{1,1}^{y}\right)>0$ and $\left(J_{1,2}^{x}+J_{1,2}^{y}\right)<0$, obtaining $|\Psi\rangle=\otimes_{l=0}^{(N / 4)-1}\left|\psi_{4 l+1}^{ \pm}\right\rangle\left|\psi_{4 l+2}^{ \pm}\right\rangle\left|\psi_{4 l+3}^{\mp}\right\rangle\left|\psi_{4 l+4}^{\mp}\right\rangle$ as a factorized ground state. This implies $J_{r, 2}^{x, y}=-\kappa J_{r, 1}^{x, y}, J_{r, 2}^{z}=\kappa J_{r, 1}^{z}, J_{r, i}^{z}$ $=\gamma_{r} J_{1, i}^{z}$, and $J_{r, i}^{x, y}=(-1)^{(r-1) / 2} \gamma_{r} J_{1, i}^{x, y}$, leading for the factorized field to $h_{F}=(1+\kappa) \sqrt{\left(\mathcal{J}_{1}+\mathcal{J}_{1}^{x}\right)\left(\mathcal{J}_{1}^{z}+\mathcal{J}_{1}^{y}\right)}$, with $\mathcal{J}_{1}^{z}=\Sigma_{r} J_{r, 1}^{z}$ and $\mathcal{J}_{1}^{x, y}=\Sigma_{r}(-1)^{(r-1) / 2} J_{r, 1}^{x, y}$

In summary, we studied the zero-temperature phase diagram of the dimerized $X Y$ chain in a transverse field. We discussed the existence of a fully unentangled ground state, which depends on whether the parameters of the system satisfy given properties. Furthermore, we showed the role of the factorizing field inside the general solution of the model. It represents a border line between two separate symmetrybroken regions in the space of the Hamiltonian parameters. In analogy with the homogeneous case, where these two regions are characterized by qualitatively different types of entanglement, namely, parallel and antiparallel entanglements, ${ }^{23}$ we expect that the same transition could take place also in our system. On the other hand, also when the Hamiltonian parameters are not compatible with groundstate factorization, there is a value of the field which separates the two regions. Finally, we extended the search for ground-state factorization to more general dimerized models.

The author gratefully acknowledges F. de Pasquale and M. Zannetti for invaluable support.
*Present address: Institute for Cross-Disciplinary Physics and Complex Systems, IFISC (CSIC-UIB), Campus Universitat Illes Balears, E-07122 Palma de Mallorca, Spain; gianluca@ifisc.uibcsic.es

${ }^{1}$ S. Sachdev, Quantum Phase Transitions (Cambridge University Press, Cambridge, 2000).

${ }^{2}$ M. Takahashi, Thermodynamics of One-Dimensional Solvable Models (Cambridge University Press, Cambridge, 1999).

${ }^{3}$ E. Lieb et al., Ann. Phys. (N.Y.) 16, 407 (1961).

${ }^{4}$ P. Pfeuty, Ann. Phys. (N.Y.) 57, 79 (1970).

${ }^{5}$ E. Barouch and B. M. McCoy, Phys. Rev. A 3, 786 (1971).

${ }^{6}$ T. J. Osborne and M. A. Nielsen, Phys. Rev. A 66, 032110 (2002).

${ }^{7}$ A. Osterloh et al., Nature (London) 416, 608 (2002).

${ }^{8}$ G. Vidal et al., Phys. Rev. Lett. 90, 227902 (2003).

${ }^{9}$ L. Amico et al., Rev. Mod. Phys. 80, 517 (2008).
${ }^{10}$ J. Kurmann et al., Physica A 112, 235 (1982).

${ }^{11}$ T. Roscilde et al., Phys. Rev. Lett. 93, 167203 (2004); 94, 147208 (2005).

${ }^{12}$ S. M. Giampaolo et al., Phys. Rev. Lett. 100, 197201 (2008).

${ }^{13}$ P. Tong and X. Liu, Phys. Rev. Lett. 97, 017201 (2006).

${ }^{14}$ J. P. de Lima et al., Phys. Rev. B 75, 214406 (2007).

${ }^{15}$ O. Derzhko et al., Phys. Rev. E 69, 066112 (2004).

${ }^{16}$ D. Shechtman et al., Phys. Rev. Lett. 53, 1951 (1984).

${ }^{17}$ R. Merlin et al., Phys. Rev. Lett. 55, 1768 (1985).

${ }^{18}$ J. H. H. Perk et al., Physica A 81, 319 (1975).

${ }^{19}$ S. Katsura, Phys. Rev. 127, 1508 (1962).

${ }^{20}$ C. Hoeger et al., J. Phys. A 18, 1813 (1985).

${ }^{21}$ R. Rossignoli et al., Phys. Rev. A 77, 052322 (2008).

${ }^{22}$ J. Kurmann et al., J. Appl. Phys. 52, 1968 (1981).

${ }^{23}$ L. Amico et al., Phys. Rev. A 74, 022322 (2006). 